\title{
Automatic Mesh Generation for 3D Smoothed Finite Element (S-FEM) based on the Weaken-weak Formulation
}

\author{
Y. $\mathrm{Li}^{1}$, J.H. Yue ${ }^{2}$, R.P. Niu ${ }^{2 *}$, and GR. Liu ${ }^{3,4}$ \\ ${ }^{1}$ Department of Mathematics, City University of Hong Kong, Hong Kong SAR, \\ China \\ ${ }^{2}$ College of Mathematics, Taiyuan University of Technology, China \\ ${ }^{3}$ Consultant, College of Mathematics, Taiyuan University of Technology, China \\ ${ }^{4}$ Department of Aerospace Engineering and Engineering Mechanics, University of \\ Cincinnati, 2851 Woodside Dr, Cincinnati OH 45221, USA
}

\begin{abstract}
The creation of smoothing domains based on a mesh of problem domain is one of the key tasks in the smoothed finite element method (S-FEM), especially for three dimensional (3D) problems. Traditionally, the smoothed strains in the 3D S-FEM implementations are calculated by using a volume-weighted average of the "raw" strains (strains in finite element method (FEM)) on the neighboring elements. In order to use the standard formulation based on the weaken-weak $\left(\mathrm{W}^{2}\right)$ formulation, we need to know not only the smoothing domains, but also the area and the outer normal vector of each surface as well as volume of smoothing domains. This paper is the first time to present a comprehensive procedure and efficient algorithms for creating smoothing domains systematically with all the necessary information required for the surface-integral needed in the smoothed strain calculations. Several algorithms are given, including two for establishing connectivity lists among nodes, edges, faces and elements and three for the generation of smoothing domains for various S-FEM models. Besides, we introduce a simple but efficient approach to determine the outer normal vector of segmental surfaces for each smoothing domain. Three examples are presented to show the effectiveness of the given algorithms.
\end{abstract}

Keywords: mesh generation, FEM, S-FEM, 3D, weaken-weak $\left(\mathrm{W}^{2}\right)$ formulation, connectivity, smoothing domains

\footnotetext{
'* ' Corresponding author.

Email addresses: yanli66-c@my.cityu.edu.hk (Y. Li), woyuejunhong@163.com (J.H. Yue),

niu ruiping2007@126.com (R.P. Niu), liugr@ucmail.uc.edu (GR. Liu)
} 


\section{Introduction}

The smoothed finite element method (S-FEM) is a powerful numerical method [2]-[4], and has been used to solve various solid mechanics problems [5]-[9]. It combines the finite element method (FEM) with some meshfree techniques using the weakened weak $\left(\mathrm{W}^{2}\right)$ formulation [10]-[12] based on the so-called G space theory [11]-[13]. The $\mathrm{W}^{2}$ form is much more general than the weak formulation and can be written in the form of Galerkin (and hence preserves the symmetry of the system) for a wide class of computational methods [14]. Compared with the weak form, $\mathrm{W}^{2}$ form reduces the consistency requirements on the assumed functions. It has been used by numerical methods for properties of upper bound, ultra-accuracy and super-convergence [14]. The essential idea in S-FEM is to modify the compatible strain field or use the strain smoothing techniques based on nodes, edges, faces and cells (elements) which leads to the node-based smoothed FEM (NS-FEM) [15][17][18], the edge-based smoothed FEM (ES-FEM) [16][19], the face-based smoothed FEM (FS-FEM) [21] and the cell-based smoothed FEM (CS-FEM) [20]. Some S-FEM models have the advantage of producing softer models and overcoming the so-called locking behavior. They offer some ways to treat the discontinuous of strain on element interfaces and improve both the displacement and the stress solution significantly [16][19]. The standard FEM gives only the lower bound solution, while the S-FEM can provide better solution with various special properties. For example, the NS-FEM can produce unique upper bound of the solutions (for force driving problems) [18], which is not possible in the standard FEM. Besides, most S-FEM models can overcome the mesh distortion issues because no mapping is required in the formulation.

The S-FEM is performed on smoothing domains based on an original mesh covering the problem domain. As the 4 node-tetrahedral (T4) mesh can be generated easily and automatically for complicated 3D geometries, they are preferred in the modeling and simulation of engineering structures in 3D problems, especially when re-mesh is needed in the process. In this paper, TetGen [36], a robust and easy-to-use software, will be used for generating T4 mesh. It generates the Delaunay and the weighted Delaunay tetrahedrons [29]-[31] for a given set of points and the constrains [32][33] for any polyhedral domain. As we know, FEM adopts the standard weak formulation, which needs differential operations upon the assumed displacement field to obtain the strain field. The S-FEM models use the smoothed Galerkin weak form, which uses only the assumed displacement and no derivative of the assumed displacement field is required. The consistency requirement on the assumed displacement function is further weakened (known as $\mathrm{W}^{2}$ formulation). However, in real 3D S-FEM implementations, the smoothed strains are usually calculated using a volume-weighted average of the "raw" strains (compatible strains) in the neighboring elements [2]. The standard integral formulation over the surface of the smoothing domains was not adopted due to the complexity of the numerical implementation. Quite an amount of information of the original mesh and the smoothing domains are required for performing the surface-integral, including the twelve connectivity lists 
among nodes, edges, faces and elements, the area and the outer normal vector of each segmental surface and the volume of smoothing domains.

In this paper, we aim to present a comprehensive process of creating smoothing domains for the family of S-FEM models based on a given original T4 mesh and the necessary information for surface integral along the surface of the smoothing domains. We have in our previous study developed a set of connectivity lists, including Ele-N, Ele-Eg, Eg-N, Eg-Ele, N-Eg and N-Ele in the previous work for 2D problems [1]. For 3D domains, we need in addition the Face-node (F-N) connectivity, Face-edge (F-Eg) connectivity, Face-element (F-Ele) connectivity, Node-face (N-F) connectivity, Edge-face (Eg-F) connectivity and Element-face (Ele-F) connectivity. These connectivity lists will be established simultaneously, which leads to less computation and time consumption. Note that there could be various ways to generate the smoothing domains. Some of these approaches, however, will spend a significant storage because the surface of one smoothing domain may be owned also by its neighboring one. In our present approach, in order to avoid duplicate storage, we choose the centroid of elements in the original mesh as the unified index, so that all the information finally is saved only once. Besides, all the surfaces of smoothing domains are remembered as triangles for the ease of calculating the area of surfaces and the volume of smoothing domains. By dividing the polyhedral smoothing domains into several tetrahedrons, the volume can be easily obtained. We claim that this work is the first time to present a comprehensive procedure and efficient algorithms for creating smoothing domains systematically with all the necessary information required for the surface-integral for smoothed strain calculation for S-FEM models.

The rest of the paper is organized as follows: Section 2 briefly introduces the FEM and the S-FEM, and then presents the standard formulation of $\mathrm{W}^{2}$ form for the S-FEM. Section 3 gives the detailed description of process and algorithms for establishing connectivity lists and creating smoothing domains for various models. Numerical results are shown in Section 4. Conclusions are provided in the final section.

\section{The idea of the S-FEM}

\subsection{Briefing on the FEM [22]-[24]}

The standard Galerkin weak form used in the FEM can be written as

$$
\int_{\Omega}(\nabla \delta \mathbf{u})^{T} \mathbf{D}(\nabla \mathbf{u}) d \Omega-\int_{\Omega} \delta \mathbf{u}^{T} \mathbf{b} d \Omega-\int_{\Gamma_{t}} \delta \mathbf{u}^{T} \mathbf{t} d \Gamma=0
$$

where $\nabla \mathbf{u}$ is the gradient of the displacement field, $\mathbf{D}$ is the matrix of material constants that is symmetric positive-definite (SPD), $\mathbf{b}$ is the vector of external body forces, $\mathbf{t}$ is the prescribed traction vector on the natural boundary, $\mathbf{u}$ and $\delta \mathbf{u}$ are trial and test function which can be expressed as:

$$
\mathbf{u}^{h}(\mathbf{x})=\sum_{I=1}^{N_{n}} \mathbf{N}_{I}(\mathbf{x}) \mathbf{d}_{I} ; \quad \delta \mathbf{u}^{h}(\mathbf{x})=\sum_{I=1}^{N_{n}} \mathbf{N}_{I}(\mathbf{x}) \delta \mathbf{d}_{I}
$$

where $N_{n}$ is the number of nodes, $\mathbf{N}_{I}(\mathbf{x})$ is the matrix of shape function and $\mathbf{d}_{I}$ is the displacement vector. 
By substituting $\mathbf{u}^{h}$ and $\delta \mathbf{u}^{h}$ into the weak form and invoking the arbitrariness of virtual nodal displacements, the standard discrete algebraic equation system of Eq.(1) is

$$
\tilde{\mathbf{K}}^{\mathrm{FEM}} \mathbf{d}=\tilde{\mathbf{f}}
$$

where $\tilde{\mathbf{K}}^{\mathrm{FEM}}$ and $\tilde{\mathbf{f}}$ are the stiffness matrix and the force vector with entries:

$$
\begin{gathered}
\tilde{\mathbf{K}}_{I J}^{\mathrm{FEM}}=\int_{\Omega} \tilde{\mathbf{B}}_{I}^{T} \mathbf{D} \tilde{\mathbf{B}}_{J} d \Omega \\
\tilde{\mathbf{f}}_{I}=\int_{\Omega} \mathbf{N}_{I}^{T}(\mathbf{x}) \mathbf{b} d \Omega+\int_{\Omega} \mathbf{N}_{\Omega}^{T}(\mathbf{x}) \mathbf{t} d \Gamma
\end{gathered}
$$

in which $\widetilde{\mathbf{B}}$ is the compatible strain gradient matrix defined as

$$
\widetilde{\mathbf{B}}_{I}(\mathbf{x})=\nabla \mathbf{N}_{I}(\mathbf{x})
$$

\subsection{The smoothed finite element method (S-FEM) [5]}

In S-FEM, we modify the compatible strain field or construct a strain field using only displacements rather than derivatives of the displacements. In particular, we choose to use the strain smoothing technique which is used to stabilize the nodal integrated meshless method [37]. The discrete set of linear system of equations is established using the smoothed Galerkin weak-form or the $\mathrm{W}^{2}$ form [10]. In order to ensure the stability, convergence and high accuracy, the modification process usually involves not only this element, but also the adjacent elements in different manners. Suitable smoothing domains, therefore, play an important role in the numerical implementation of S-FEM. The key technique of S-FEM, strain smoothing operation can then perform on the obtained smoothing domains by surface-integral. Therefore, there is no need to do 3D volume integration when we use the $\mathrm{W}^{2}$ form.

Using the strain smoothing operation, the compatible strain works for creating smoothed strain on the smoothing domain $\Omega^{(k)}$.

$$
\overline{\boldsymbol{\varepsilon}}^{(k)}=\int_{\Omega^{(k)}} \widetilde{\boldsymbol{\varepsilon}}(\mathbf{x}) \Phi^{(k)}(\mathbf{x}) d \Omega=\int_{\Omega^{(k)}} \nabla \mathbf{u}(\mathbf{x}) \Phi^{(k)}(\mathbf{x}) d \Omega
$$

where $\Phi^{(k)}(\mathbf{x})$ is a given smoothing function that satisfies at least unity property

$$
\int_{\Omega^{(k)}} \Phi^{(k)}(\mathbf{x}) d \Omega=1
$$

The general formulation for computing the smoothed stiffness matrix is

$$
\overline{\mathbf{K}}=\int_{\Omega} \overline{\mathbf{B}}^{\mathrm{T}} \mathbf{D} \overline{\mathbf{B}} d \Omega
$$

with entries

$$
\overline{\mathbf{K}}_{I J}=\sum_{k=1}^{N_{s}} V^{(k)} \overline{\mathbf{B}}_{I}^{T} \mathbf{D} \overline{\mathbf{B}}_{J}
$$

Note that only the volume and the usual compatible strain gradient matrices of elements are required when we calculate the system stiffness matrix with this formulation. Using the divergence theorem, the smoothed strain matrix $\overline{\mathbf{B}}_{I}\left(\mathbf{x}_{k}\right)$ on the domain $\Omega^{(k)}$ can be calculated by 


$$
\overline{\mathbf{B}}_{I}\left(\mathbf{x}_{k}\right)=\left[\begin{array}{ccc}
\bar{\phi}_{I x}\left(\mathbf{x}_{k}\right) & 0 & 0 \\
0 & \bar{\phi}_{I y}\left(\mathbf{x}_{k}\right) & 0 \\
0 & 0 & \bar{\phi}_{I I}\left(\mathbf{x}_{k}\right) \\
\bar{\phi}_{I y}\left(\mathbf{x}_{k}\right) & \bar{\phi}_{I x}\left(\mathbf{x}_{k}\right) & 0 \\
0 & \bar{\phi}_{I z}\left(\mathbf{x}_{k}\right) & \bar{\phi}_{I y}\left(\mathbf{x}_{k}\right) \\
\bar{\phi}_{I z}\left(\mathbf{x}_{k}\right) & 0 & \bar{\phi}_{I x}\left(\mathbf{x}_{k}\right)
\end{array}\right]
$$

with

$$
\bar{\phi}_{I l}=\frac{1}{V^{(k)}} \sum_{m=1}^{n_{s e g}}\left[\sum_{n=1}^{n_{G}} W_{n}^{G}\left(\phi_{I}\left(\mathbf{x}_{m, n}\right) n_{l, m}\right) A_{I}^{(k)}\right] \quad(l=x, y, z)
$$

in which $n_{\text {seg }}$ denotes the number of surfaces of smoothing domain and $n_{G}$ is the number of Gauss points located within each surface. $W_{n}^{G}$ is the weight value, $n_{l, m}$ is the l-th component of the outer normal vector on the m-th segmental surface and $A_{I}^{(k)}$ is the area of the surface.

It can be seen from Eq.(13) that we need only the shape function values at some particular points on the smoothing doma in surfaces. This gives much more freedom for constructing shape function. Besides, it is straightforward to extend the S-FEM for higher-order interpolation schemes while calculating the smoothed strain matrix using Eq.(12).

\section{The process for creating smoothing domains}

\section{1 Element-connectivity required in S-FEM models}

The S-FEM models (CS-, ES-, FS- and NS-FEM) for 3D problems work well with a set of background T4 elements and a proper connectivity. In our work [5], we use the software TetGen to generate T4 mesh before the construction of smoothing domains. For creating smoothing domains, twelve connectivity lists among nodes, edges, faces and elements are required. Except the six connectivity lists of Element-node (Ele-N), Element-edge (Ele-Eg), Edge-node (Eg-N), Edge-element (Eg-Ele) and Node-edge (N-Eg), Node-element (N-Ele) which have been introduced in the previous work for 2D problems [1], we need also the following six connectivity lists.

1) Face-node (F-N) connectivity: records the nodes owned by each face. It is the basic requirement for all the S-FEM models.

2) Face-edge (F-Eg) connectivity: records the edges owned by each face. It is required for creating smoothing domains on the boundary of the problem domain.

3) Face-element (F-Ele) connectivity: records elements associated with each face. An inner face connects to two elements while a boundary face belongs to only one. It is used for constructing smoothing domains on the boundary.

4) Node-face (N-F) connectivity: records the faces connected directly to each node. The number of faces connected to one node usually different from that to another node. 
5) Edge-face (Eg-F) connectivity: records the faces connected to each edge. The number of faces connected to one edge may also be different from that to another edge. It is used for ES-FEM model.

6) Element-face (Ele-F) connectivity: records the faces of each element. It is essential for all the S-FEM models.

\section{2 Algorithms for the creation of smoothing domains}

The S-FEM models are created based on cells (elements), faces, edges and nodes on the original background mesh. For ease of implementation, we will establish all the necessary connectivity first. However, not all the connectivity is required for a certain S-FEM model. The general process of creating smoothing domains can be summarized as:

Step.1: Generate quality T4 mesh using TetGen with outputs of coordinates of nodes and three connectivity lists Eg-N, F-N and Ele-N.

Step.2: Build connectivity lists N-Eg, N-F, N-Ele, Eg-F, Eg-Ele, F-Eg, F-Ele, Ele-Eg, Ele-F based on the given results. Some of the connectivity can be established at the same time.

Step.3: Calculate the centroid of each element, the center of each face and the midpoint of each edge. Remember these newly generated points and establish the corresponding N-Ele connectivity.

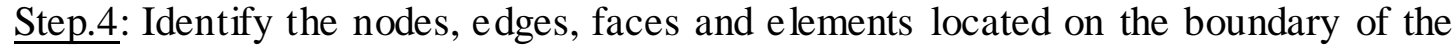
problem domain.

Step.5: Create smoothing domains for ES-, FS-, NS-FEM models. Each smoothing domain is a polyhedron. Some surfaces of the polyhedron are owned by more than one smoothing domains. Save the surfaces and smoothing domains as well as the relationship between them.

Step.6: Calculate the area and the outer normal vector of each surface and the volume of each smoothing domain.

Note that the outer normal vector of surface for different smoothing domains will be different. Therefore, we will first calculate the area and the normal vector when a new surface is created. The direction of the outer normal vector will be determined later when smoothing domains are created.

\section{3 Algorithm for generating the connectivity lists}

\subsection{Building the connectivity of N-Ele, N-Eg and N-F}

For a T4 mesh, one element is associated with one Ele-N connectivity, four N-Ele connectivity, four F-N connectivity, four N-F connectivity, six Eg-N connectivity and four N-Eg connectivity. Based on the numbered elements, faces, edges and nodes as well as the Ele-N, F-N and Eg-N connectivity given in the output file of TetGen, we can easily establish the connectivity lists of N-Ele, N-F and N-Eg. Here we introduce only the algorithm for building the N-Ele connectivity, the connectivity of N-F and $\mathrm{N}$-Eg can be obtained in a similar way. 


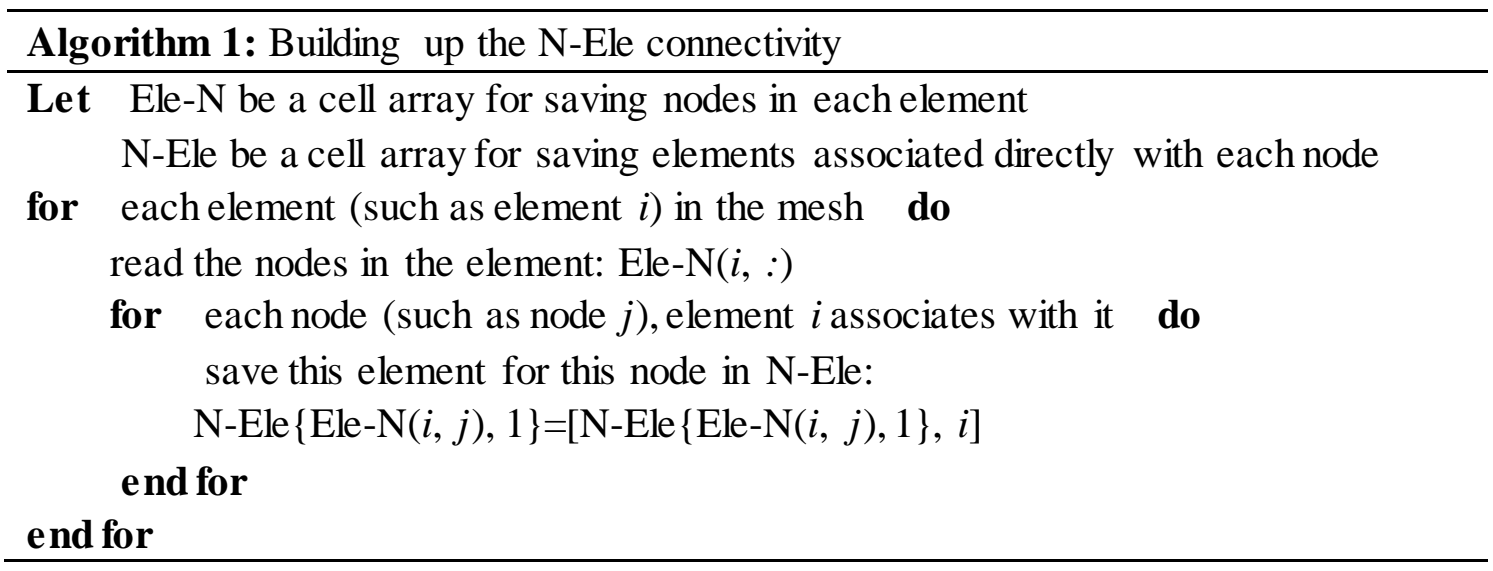

\subsection{Building the connectivity lis ts of Eg-Ele, Ele-Eg, Eg-F, F-Eg, F-Ele and}

Ele-F

Contrary connectivity can be built at the same time which means Ele-Eg connectivity can be established while building the Eg-Ele connectivity, Eg-F connectivity can be established while building the F-Eg connectivity, F-Ele connectivity can be established while building the Ele-F connectivity. One element contains four edges and each edge has two endpoints. The Eg-Ele connectivity can be established based on the obtained N-Ele connectivity and the Eg-N connectivity which has been given in the output file of the original mesh.

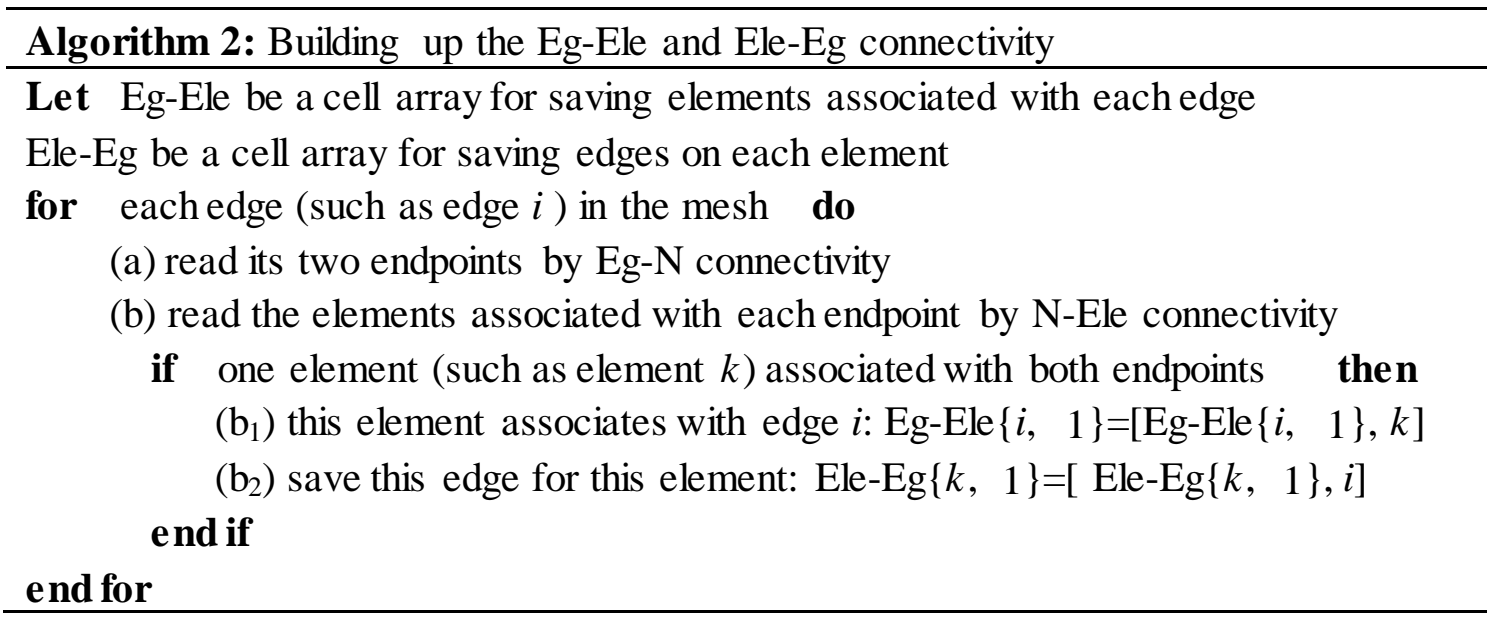

The Eg-F and F-Eg connectivity, F-Ele and Ele-F connectivity can be established in a similar way. Using N-F connectivity instead of N-Ele connectivity in step (b), the Eg-F and the F-Eg connectivity can be established. The connectivity of F-Ele and Ele-F can be built up by changing Eg-N connectivity in step (a) to F-N connectivity.

\subsection{Identify the nodes, edges, faces and elements on the boundary}

TetGen, the software we used for T4 mesh generation, will produce tags which are an integer to identify faces on the boundary of the problem domain. If the boundary marker is 1 , it means this face locates on the boundary. Otherwise the marker is 0 . Obviously, nodes and edges on the boundary face are on the boundary of the problem 
domain also. Elements associated with any of these faces are on the boundary.

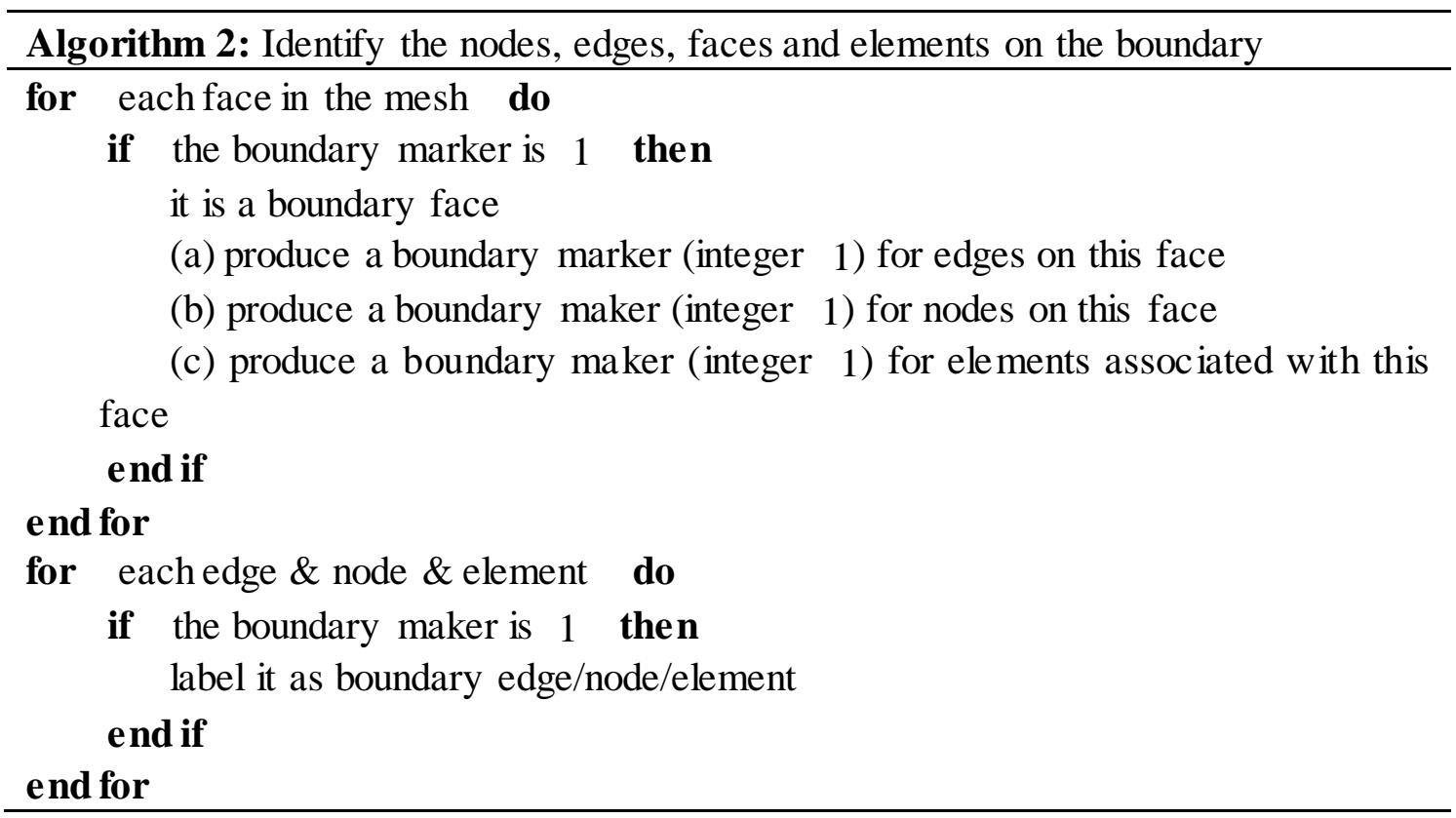

\section{1 The creation of smoothing domains for S-FEM models}

In S-FEM, the problem domain should be divided into a set of 'non-overlap' and 'no-gap' smoothing domains covering the original tetrahedral mesh. In this section we will introduce algorithms for creating smoothing domains for NS-FEM, ES-FEM and FS-FEM models.

\subsection{Creating node-based smoothing domain}

For NS-FEM, the smoothing domains are formed associated with nodes in the mesh. Actually, the smoothing domain for a certain node consists of portions from all T4 elements sharing this node. As shown in Figure 1 , this element with vertex $\mathrm{N}_{1}, \mathrm{~N}_{2}, \mathrm{~N}_{3}$ and $\mathrm{N}_{4}$ will contribute equally to four smoothing domains based on each vertex. Surfaces of the smoothing domains are formed by the mid-point of edges (A-F), the center of faces(G-J) and the centroid of element (K). It is evident that $\mathrm{N}_{1}$-based smoothing domain from element $i$ is formed by connecting points D-J-K-H-C-G-A with triangular faces DJK, DHK, AGK, AHK, CJK and CGK. Therefore, the complete smoothing domain based on $\mathrm{N}_{1}$ can be obtained when we do the same work on all the elements associated with it.

Algorithm 3: Creating the node-based smoothing domain

for each T4 element in the original mesh do

(a) establish the local Eg-F connectivity in the element

(b) create smoothing domains and remember their surfaces

for each edge in the element do

(b1) read the faces in this element associated with this edge by local Eg-F connectivity 
(b2) read the midpoint of this edge and the center of the faces given in (b1)

(b3) remember the surfaces formed by the midpoint, the centroid of this element and the center of each surface

(b4) record the surfaces in the smoothing domains based on each endpoint of this edge

\section{end for}

\section{end for}

for all the faces on the boundary of the problem domain do

(a) establish the local N-Eg connectivity in this face

for each vertex in the face do

(b1) save the two triangular surfaces cons ist of vertex, the center of face and the midpoint of each edge associated with this vertex (by the local N-Eg connectivity)

(b2) remember the two surfaces in the smoothing domain based on this vertex.

\section{end for}

\section{end for}

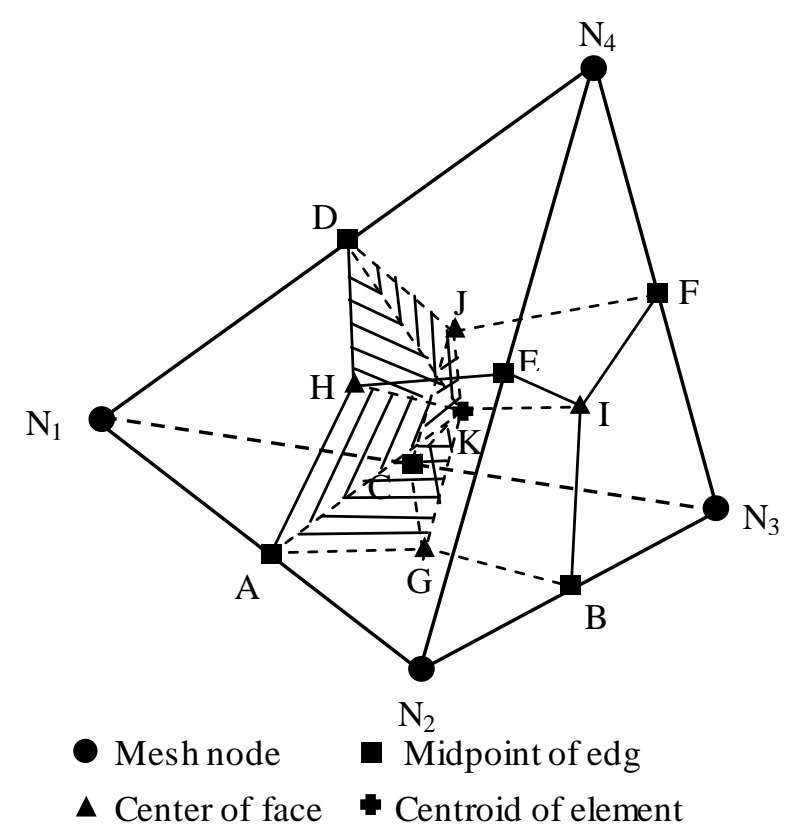

Figure 1: The node-based smoothing domains contributed from one T4 elements.

\subsection{Creating edge-based smoothing domain}

In ES-FEM model, T4 mesh will be re-divided according to edges of elements, hence the number of smoothing domains equals to the total number of edges. It is larger than the required minimum number of smoothing domains for a stable S-FEM model. In $3 \mathrm{D}$ case the edge-based smoothing domains are enclosed by a couple of surfaces who are formed by nodes of elements, the center of face and the centroid of element. It can be seen from Figure 2, one T4 element contributes equally to six smoothing domains 
based on each edge of this element. These edge-based smoothing domains can be created respectively by using the edges as indexes. A better choice is adopting the faces on the element as indexes. Connecting the center of a certain face and the centroid of element with each of the vertex, three surfaces of smoothing domains are formed. By establishing the local N-Eg connectivity for each vertex in this face, we get to know the formed surfaces are owned by which smoothing domains. For example, we can obta in three triangular surfaces based on the face $\mathrm{N}_{1} \mathrm{~N}_{2} \mathrm{~N}_{4}$, they are $A_{C N}, A_{2}, A C N_{4}$. By the local N-Eg connectivity in the face we know $N_{2}$ associates with edge $\mathrm{N}_{1} \mathrm{~N}_{2}$ and $\mathrm{N}_{2} \mathrm{~N}_{4}$. Then the surface $A C \mathrm{~N}_{2}$ who contains node $\mathrm{N}_{2}$ is owned by $\mathrm{N}_{1} \mathrm{~N}_{2}$-based and $\mathrm{N}_{2} \mathrm{~N}_{4}$-based smoothing domains. Similarly surface $\mathrm{ACN}_{4}$ is owned by $\mathrm{N}_{2} \mathrm{~N}_{4}$-based and $\mathrm{N}_{1} \mathrm{~N}_{4}$-based smoothing domains, surface $\mathrm{ACN}_{1}$ is owned by $\mathrm{N}_{1} \mathrm{~N}_{2}$-based and $\mathrm{N}_{1} \mathrm{~N}_{4}$-based smoothing domains. Using faces as indexes to create the surfaces of smoothing domains can avoid duplicate storage. There are quite a lot of such triangular surfaces in 3D smoothing domains, this approach can have a significant reduction in memory consumption.

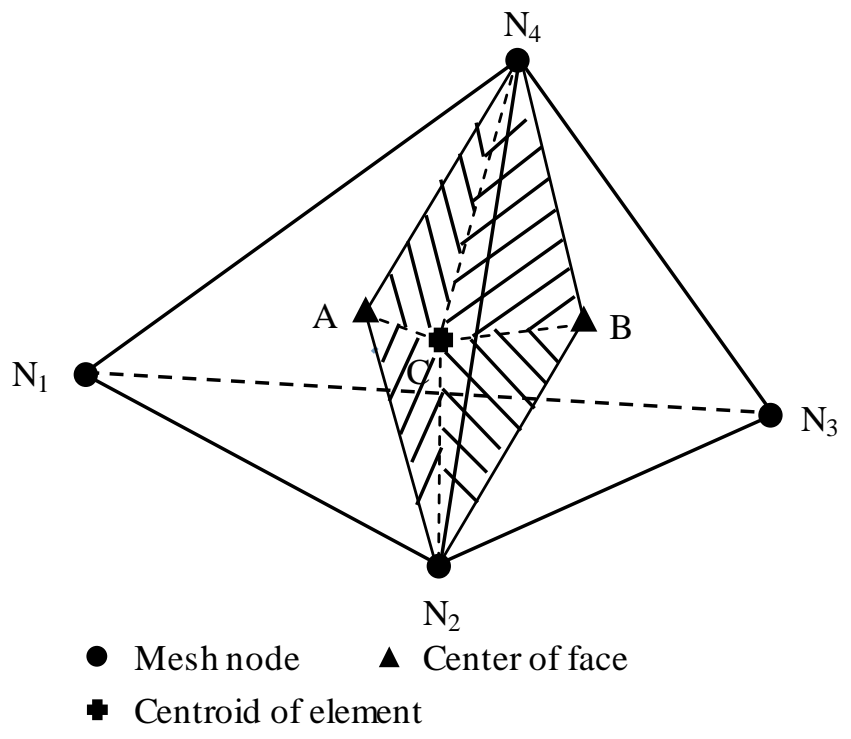

Figure 2: The edge-based smoothing domains contributed from one T4 element

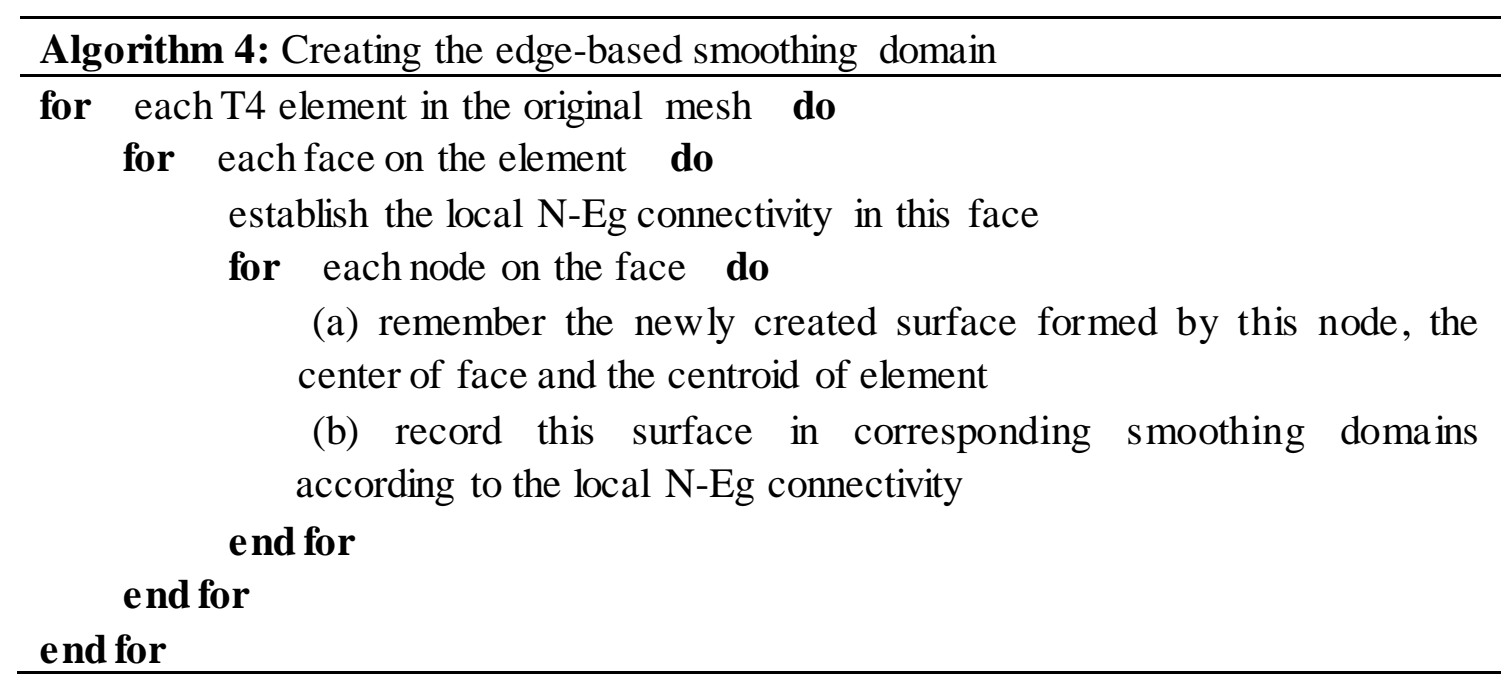


for each face on the boundary of the problem domain do

for each edge on the face do

(a) remember the triangle formed by two endpoints of this edge and the center of face as surface

(b) record this triangular in the surface lists of the smoothing domain based on this edge

end for

end for

\subsection{Creating face-based smoothing domains}

In FS-FEM, smoothing domains are created by connecting nodes on the face with the centroid of adjacent elements. For an inner face, as shown in Figure 3, the smoothing domain based on face $\mathrm{N}_{1} \mathrm{~N}_{2} \mathrm{~N}_{3}$ is constructed by connecting centroids $C_{i}$ and $C_{j}$ with the three vertex of the triangle $\left(\mathrm{N}_{1}, \mathrm{~N}_{2}\right.$ and $\left.\mathrm{N}_{3}\right)$. This face-based smoothing domain is a hexahedron. If the face locates on the boundary of the problem domain, the smoothing domain is a tetrahedron formed by connecting the centroid of the host tetrahedron element and the three vertexes on this face.

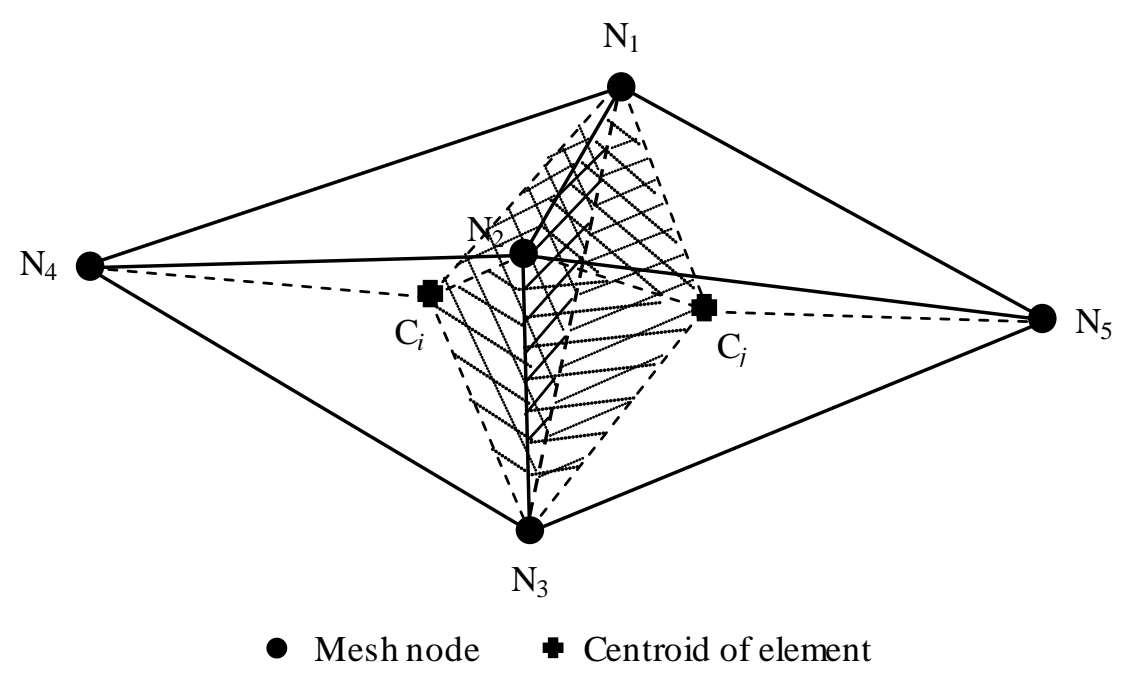

Figure 3: The face-based smoothing domain for an inner face

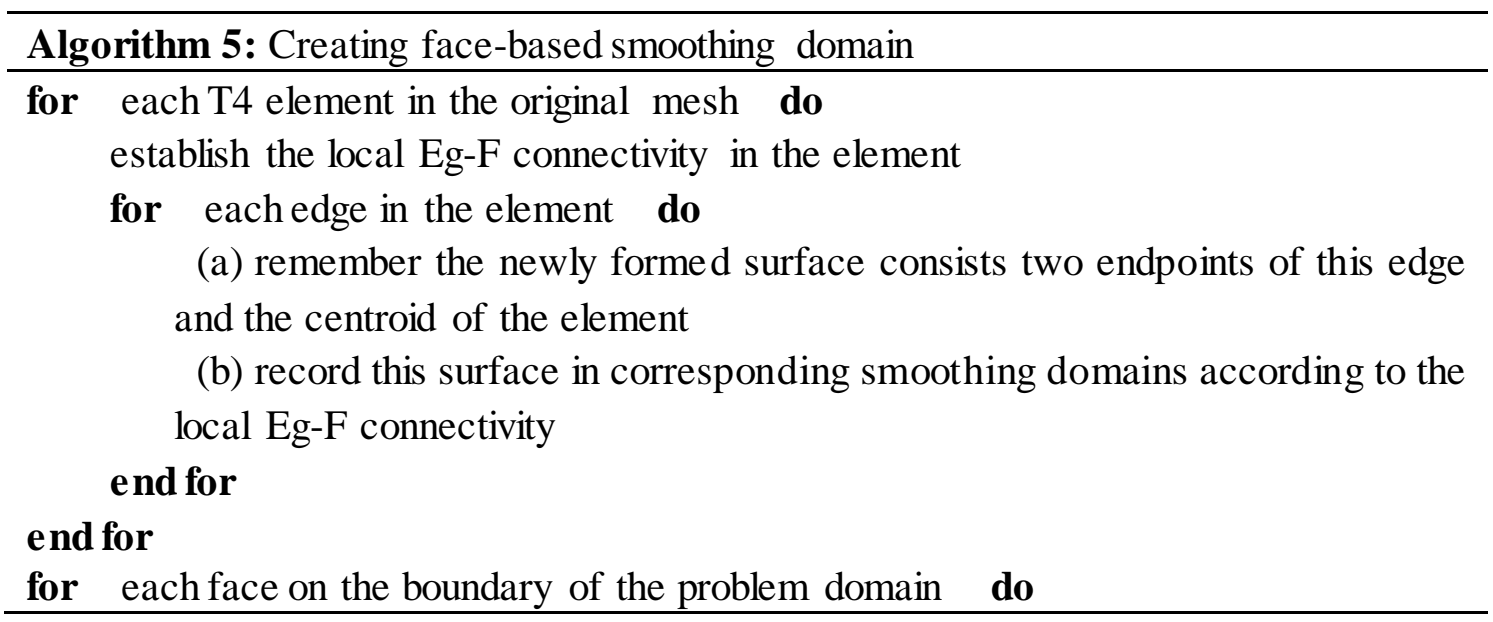


(a) remember the face itself as a surface

(b) record this surface in the smoothing domain based on itself

end for

\subsection{Calculation of area and the outer normal vector of surfaces}

Instead of approximating the strain using the simple average of the compatible strain based on the volume of smoothing domains [25]-[27], the strain gradient matrix in this paper (see Eq. (12)) is evaluated by doing the integration along the surfaces of smoothing domains which can be carried out using Gauss quadrature technique [25]. It can be seen from Eq. (12) that the area and the outer normal vector of surfaces are required for surface integral. The area and the normal vector of a triangular surface can be easily calculated with coordinates of the vertexes. However, the outer normal vector of one surface is not unique. It may be in opposite direction when we do integral on this surface for different smoothing domains.

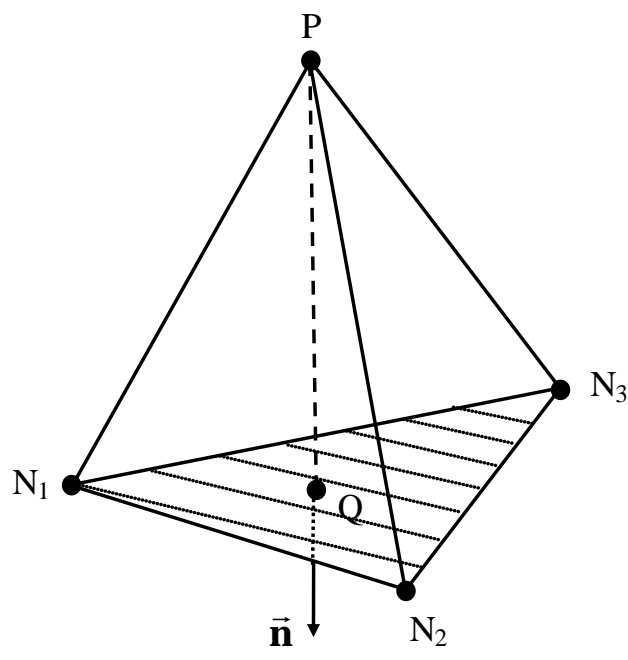

Figure 4: One part of a smoothing domain: $\mathrm{N}_{1} \mathrm{~N}_{2} \mathrm{~N}_{3}$ is one surface and $\mathrm{P}$ is an inside point.

Assume $\mathrm{N}_{1} \mathrm{~N}_{2} \mathrm{~N}_{3}$ be one surface of a smoothing domain with an inside point P. The coordinates of points $\mathrm{N}_{1}, \mathrm{~N}_{2}$ and $\mathrm{N}_{3}$ are $\left(x_{1}, y_{1}, z_{1}\right),\left(x_{2}, y_{2}, z_{2}\right),\left(x_{3}, y_{3}, z_{3}\right)$ The normal vector $\overrightarrow{\mathbf{n}}$ and the area $A$ of triangular surface $N_{1} N_{2} N_{3}$ can be calculated by the following formulas:

$$
\begin{gathered}
\overrightarrow{\mathbf{n}}=\left(n_{1}, n_{2}, n_{3}\right)=\left(x_{1}-x_{2}, y_{1}-y_{2}, z_{1}-z_{2}\right) \times\left(x_{1}-x_{3}, y_{1}-y_{3}, z_{1}-z_{3}\right) \\
\mathrm{A}=|\overrightarrow{\mathbf{n}}| / 2
\end{gathered}
$$

The equation of the plane which contains $\mathrm{N}_{1} \mathrm{~N}_{2} \mathrm{~N}_{3}$ can be obtained based on the normal vector and a known point (such as $\mathrm{N}_{1}$ ) on the plane:

$$
n_{1} x+n_{2} y+n_{3} z+d=0
$$

where $d=-\left(n_{1} x_{1}+n_{2} y_{1}+n_{3} z_{1}\right)$. The normal vector can be positive or negative. 
However, the outer normal vector of the specific surface for a certain smoothing domain is unique. We have to judge the direction of normal vectors each time when we use Eq.(12) to compute the strain gradient matrix, which is a time consuming process. A better way to do this is by computation rather than judging which will be more efficient and be helpful for parallel computing. In Figure $4, \mathrm{~N}_{1} \mathrm{~N}_{2} \mathrm{~N}_{3}$ is the surface of one smoothing domain (such as $\Omega_{k}^{s}$ ) with center point P. When computing the outer normal vector of surface $\mathrm{N}_{1} \mathrm{~N}_{2} \mathrm{~N}_{3}$ associated with smoothing domain $\Omega_{k}^{s}$, we select a point inside the smoothing domain (such as node $\mathrm{P}$ with coordinates $\left.\left(p_{1}, p_{2}, p_{3}\right)\right)$ and find its projection (point $\mathrm{Q}$ ) on the surface. The direction of the outer normal vector and the vector $\overrightarrow{P Q}$ should be the same. Then the coordinate of point $\mathrm{Q}$ (assume to be $(x, y, z))$ should satisfy two conditions: the vector $\overrightarrow{P Q}$ parallels the normal vector $\overrightarrow{\mathbf{n}}$ and point $\mathrm{Q}$ locates on the shadow surface. That is:

$$
\left\{\begin{array}{l}
\frac{x-p_{1}}{n_{1}}=\frac{y-p_{2}}{n_{2}}=\frac{z-p_{3}}{n_{3}}=k \\
n_{1} x+n_{2} y+n_{3} z+d=0
\end{array}\right.
$$

where $\left(x-p_{1}, y-p_{2}, z-p_{3}\right)$ is the outer normal vector and $k$ is a constant. Solving the resulting equations simultaneously, we have

$$
k=-\frac{d+n_{1} p_{1}+n_{2} p_{2}+n_{3} p_{3}}{n_{1}^{2}+n_{2}^{2}+n_{3}^{2}}
$$

Therefore, the outer normal vector can be easily obtained by multiplying the normal vector by a constant.

$$
\overrightarrow{\mathbf{n}}_{\text {out }}=\overrightarrow{\mathbf{n}} \cdot k
$$

\subsection{Calculation of the volume of each smoothing domain}

The smoothing domains of various S-FEM models are always polyhedron whose volume can hardly be calculated by a standard formula. As we have remembered the surfaces of smoothing domains as triangular, we can divide the smoothing domain into several tetrahedrons whose volume can be easily obtained. Connecting the vertexes of each surface with one point inside the smoothing domain, the smoothing domain is partitioned into a couple of non-over and non-gap tetrahedrons. The location of this inside point is random, but it must be the same for all the surfaces on one smoothing domain. For instance, we can use the center of face for FS-FEM, the mid-point of edge for ES-FEM and the node itself for NS-FEM.

\section{Test examples}

In order to demonstrate the effectiveness of the given algorithms, we do numerical tests on 3D problems and present twelve connectivity lists and information of various smoothing domains. 


\section{1 Example 1: A simple domain with two T4 elements}

In order to show clearly the procedure of our algorithm for creating smoothing domains, we choose a simple geometry which cons ists of two T4 elements as the first example. Input the coordinates of points at vertexes of the problem domain (Figure 5), the TetGen automatically generates all the nodes, edges, faces and elements in the mesh as well the connectivity of Ele-N, F-N and Eg-N. Then we will establish the other nine connectivity lists by Algorithm 1 and 2. Table 1-Table 4 lists the connectivity re lated to each node, edge, face, and element. The smoothing doma ins on the boundary of the problem domain may have different features with those inside ones. We add a boundary marker to separate those items on the boundary (Algorithm 3 ). For those located on the boundary, we assign the marker 1 , otherwise the marker 0 is assigned. In Table 2-Table 4, we present als o the centroid of elements, the center of faces and the midpoint of edges which are required during the smoothing doma in creation process.

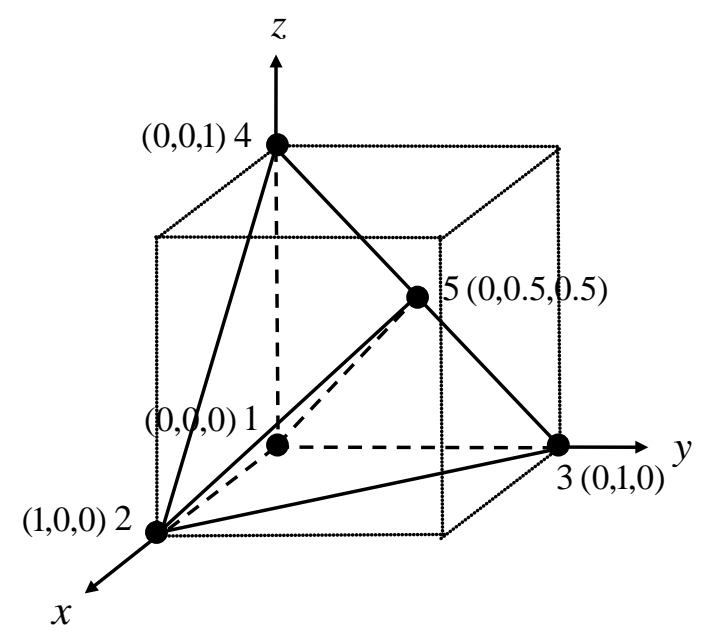

Figure 6: A simple domain with two T4 elements

Table 1: Connectivity lists and boundary marker for nodes.

\begin{tabular}{c|c|c|c|c}
\hline Node No. & N-Eg & N-F & N-Ele & Boundary marker \\
\hline 1 & $2-3-6-8$ & $2-3-4-6-7$ & $1-2$ & 1 \\
2 & $1-4-6-9$ & $1-2-4-5-7$ & $1-2$ & 1 \\
3 & $7-8-9$ & $5-6-7$ & 2 & 1 \\
4 & $3-4-5$ & $1-3-4$ & 1 & 1 \\
5 & $1-2-5-7$ & $1-2-3-5-6$ & $1-2$ & 1 \\
\hline
\end{tabular}

Table 2: Connectivity lists boundary marker for elements.

\begin{tabular}{c|c|c|c|c|c}
\hline Element No. & Ele-N & Ele-Eg & Ele-F & Centroid & Boundary marker \\
\hline 1 & $1-4-2-5$ & $1-2-3-4-5-6$ & $1-2-3-4$ & 6 & 1 \\
2 & $3-1-2-5$ & $1-2-6-7-8-9$ & $2-5-6-7$ & 7 & 1 \\
\hline
\end{tabular}


Table 3: Connectivity lists boundary marker for faces.

\begin{tabular}{c|c|c|c|c|c}
\hline Face No. & F-N & F-Eg & F-Ele & Center & Boundary marker \\
\hline 1 & $5-2-4$ & $1-4-5$ & 1 & 8 & 1 \\
2 & $5-1-2$ & $1-2-6$ & $1-2$ & 9 & 0 \\
3 & $4-1-5$ & $2-3-5$ & 1 & 10 & 1 \\
4 & $4-2-1$ & $3-4-6$ & 1 & 11 & 1 \\
5 & $5-3-2$ & $1-7-9$ & 2 & 12 & 1 \\
6 & $1-3-5$ & $2-7-8$ & 2 & 13 & 1 \\
7 & $1-2-3$ & $6-8-9$ & 2 & 14 & 1 \\
\hline
\end{tabular}

Table 4: Connectivity lists boundary marker for edges.

\begin{tabular}{c|c|c|c|c|c}
\hline Edge No. & Eg-N & Eg-F & Eg-Ele & Midpoint & Boundary marker \\
\hline 1 & $5-2$ & $1-2-5$ & $1-2$ & 15 & 1 \\
2 & $5-1$ & $2-3-6$ & $1-2$ & 16 & 1 \\
3 & $4-1$ & $3-4$ & 1 & 17 & 1 \\
4 & $4-2$ & $1-4$ & 1 & 18 & 1 \\
5 & $4-5$ & $1-3$ & 1 & 19 & 1 \\
6 & $1-2$ & $2-4-7$ & $1-2$ & 20 & 1 \\
7 & $5-3$ & $5-6$ & 2 & 21 & 1 \\
8 & $1-3$ & $6-7$ & 2 & 22 & 1 \\
9 & $3-2$ & $5-7$ & 2 & 23 & 1 \\
\hline
\end{tabular}

By connecting nodes on the surface with the centroid of neighboring elements, face-based smoothing domains are constructed (Figure 1). Table 5 presents information of the triangular surfaces of the face-based smoothing domains. The edge-based smoothing domain is created by connecting the centroid of elements and the center of faces associated with each edge and its two endpoints (Figure 2). In Table 7 we give information of the surfaces of edge-based smoothing domains. When we connect the centroid of elements, the center of faces and the midpoint of edges associated with each node in the mesh, we establish the smoothing domains based on nodes (Figure 3). The information of surfaces of these smoothing domains is shown in Table 9. All the smoothing domains are polyhedron which consists of several triangular surfaces, for instance, a smoothing domain based on a face inside the problem domain has six triangular surfaces (Figure 3). The area and the outer normal vector which are necessary for doing surface-integral in W2 formulation can be calculated with vertexes on the surface. Each time when a new surface is generated, we compute its area and normal vector. The values have been shown in Table 5, Table 7 and Table 9 respectively. Although some surfaces may be owned by different smoothing domains, all the surfaces will be saved for only once for saving the memory consumption. In Table 6, Table 8 and Table 10, we show a smoothing doma in is formed by which surfaces. As we have introduced in Equation (19), the outer normal vector can be obtained by multiplying the normal vector by a constant, positive or negative. If it is positive, as shown in Table 6, Table 8 and Table 10, the outer normal vector of the surface is right the normal vector given in Table5, Table 7 
and Table 9, otherwise it is in the opposite direction to the normal vector we computed. Besides, the volume of smoothing domains which are required for W2 formulation is also given in Table 6, Table 8 and Table 10.

Table 5: The triangular surfaces of smoothing domains in face-based smoothing domains
\begin{tabular}{c|c|c|c|c|c|c|c} 
No. & Vertex & Area & Normal vector & No. & Vertex & Area & Normal vector \\
\hline 1 & $6-5-2$ & 0.1398 & $(-0.45,0,-0.89)$ & 10 & $7-5-3$ & 0.1531 & $(-0.82,-0.41,-0.41)$ \\
2 & $6-5-1$ & 0.1083 & $(-0.58,-0.58,0.58)$ & 11 & $7-1-3$ & 0.1398 & $(0.45,0,-0.89)$ \\
3 & $6-4-1$ & 0.1398 & $(0.45,-0.89,0)$ & 12 & $7-3-2$ & 0.2073 & $(-0.30,-0.30,-0.90)$ \\
4 & $6-4-2$ & 0.2073 & $(0.30,0.90,0.30)$ & 13 & $5-2-4$ & 0.4330 & $(-0.58,-0.58,-0.58)$ \\
5 & $6-4-5$ & 0.1531 & $(-0.82,-0.41,-0.41)$ & 14 & $4-1-5$ & 0.2500 & $(1,0,0)$ \\
6 & $6-1-2$ & 0.1976 & $(0,-0.95,0.32)$ & 15 & $4-2-1$ & 0.5000 & $(0,1,0)$ \\
7 & $7-5-2$ & 0.1398 & $(0.45,0.89,0)$ & 16 & $5-3-2$ & 0.4330 & $(-0.58,-0.58,-0.58)$ \\
8 & $7-5-1$ & 0.1083 & $(0.58,-0.58,0.58)$ & 17 & $1-3-5$ & 0.2500 & $(1,0,0)$ \\
9 & $7-1-2$ & 0.1976 & $(0,-0.32,0.95)$ & 18 & $1-2-3$ & 0.5000 & $(0,0,1)$ \\
\hline
\end{tabular}

Table 6: The face-based smoothing domains

\begin{tabular}{c|c|c|c}
\hline No. & Surfaces & Outer normal vector direction & Volume \\
\hline 1 & $1,4,5,13$ & $1,-1,1,-1$ & 0.0208 \\
2 & $1,2,6,7,8,9$ & $-1,1,1,1,-1,-1$ & 0.0417 \\
3 & $2,3,5,14$ & $-1,1,-1,-1$ & 0.0208 \\
4 & $3,4,6,15$ & $-1,1,-1,-1$ & 0.0208 \\
5 & $7,10,12,16$ & $-1,1,1,-1$ & 0.0208 \\
6 & $8,10,11,17$ & $1,-1,1,-1$ & 0.0208 \\
7 & $9,11,12,18$ & $1,-1,-1,-1$ & 0.0208 \\
\hline
\end{tabular}

Table 7: The triangular surfaces of smoothing domains in edge-based smoothing domains

\begin{tabular}{c|c|c|c|c|c|c|c}
\hline No. & Vertex & Area & Normal vector & No. & Vertex & Area & Normal vector \\
\hline 1 & $6-8-5$ & 0.0361 & $(-0.58,-0.58,0.58)$ & 22 & $7-14-1$ & 0.0361 & $(-0.58,0.58,-0.58)$ \\
2 & $6-8-2$ & 0.0659 & $(0,0.95,-0.32)$ & 23 & $7-14-2$ & 0.0466 & $(-0.45,-0.89,0)$ \\
3 & $6-8-4$ & 0.0466 & $(0.45,-0.89,09)$ & 24 & $7-14-3$ & 0.0510 & $(0.82,0.41,0.41)$ \\
4 & $6-9-5$ & 0.0510 & $(0.82,0.41,0.41)$ & 25 & $5-2-8$ & 0.1443 & $(-0.58,-0.58,-0.58)$ \\
5 & $6-9-1$ & 0.0466 & $(-0.45,0.89,0)$ & 26 & $4-2-8$ & 0.1443 & $(0.58,0.58,0.58)$ \\
6 & $6-9-2$ & 0.0691 & $(-0.30,-0.90,-0.30)$ & 27 & $4-5-8$ & 0.1443 & $(-0.58,-0.58,-0.58)$ \\
7 & $6-10-4$ & 0.0691 & $(0.30,0.90,0.30)$ & 28 & $5-1-10$ & 0.0833 & $(-1,0,0)$ \\
8 & $6-10-1$ & 0.0659 & $(0,-0.95,0.32)$ & 29 & $4-1-10$ & 0.0833 & $(1,0,0)$ \\
9 & $6-10-5$ & 0.0466 & $(-0.45,0,-0.89)$ & 30 & $4-5-10$ & 0.0833 & $(-1,0,0)$ \\
10 & $6-11-4$ & 0.0510 & $(-0.82,-0.41,-0.41)$ & 31 & $4-1-11$ & 0.1667 & $(0,-1,0)$ \\
11 & $6-11-2$ & 0.0466 & $(0.45,0,0.89)$ & 32 & $4-2-11$ & 0.1667 & $(0,1,0)$ \\
12 & $6-11-1$ & 0.0361 & $(0.58,0.58,-0.58)$ & 33 & $1-2-11$ & 0.1667 & $(0,-1,0)$ \\
13 & $7-9-5$ & 0.0510 & $(-0.82,-0.41,-0.41)$ & 34 & $5-2-12$ & 0.1443 & $(0.58,0.58,0.58)$ \\
14 & $7-9-1$ & 0.0466 & $(0.45,0,-0.89)$ & 35 & $5-3-12$ & 0.1443 & $(-0.58,-0.58,-0.58)$ \\
15 & $7-9-2$ & 0.0691 & $(0.30,0.30,0.90)$ & 36 & $3-2-12$ & 0.1443 & $(-0.58,-0.58,-0.58)$ \\
16 & $7-12-5$ & 0.0361 & $(0.58,-0.58,0.58)$ & 37 & $5-1-13$ & 0.0833 & $(1,0,0)$
\end{tabular}




\begin{tabular}{l|c|c|c|c|c|c|c}
17 & $7-12-3$ & 0.0466 & $(-0.45,0,0.89)$ & 38 & $5-3-13$ & 0.0833 & $(-1,0,0)$ \\
18 & $7-12-2$ & 0.0659 & $(0,0.32,-0.95)$ & 39 & $1-3-13$ & 0.0833 & $(1,0,0)$ \\
19 & $7-13-1$ & 0.0659 & $(0,-0.32,0.95)$ & 40 & $1-2-14$ & 0.1667 & $(0,0,1)$ \\
20 & $7-13-3$ & 0.0691 & $(-0.30,-0.30,-0.90)$ & 41 & $1-3-14$ & 0.1667 & $(0,0,-1)$ \\
21 & $7-13-5$ & 0.0466 & $(0.45,0.89,0)$ & 42 & $3-2-14$ & 0.1667 & $(0,0,-1)$ \\
\hline
\end{tabular}

Table 8: The edge-based smoothing domains

\begin{tabular}{c|c|c|c}
\hline No. & Surfaces & Outer normal vector & Volume \\
\hline 1 & $1,2,4,6,13,15,16,18,25,34$ & $1,-1,-1,1,1,-1,-1,1,-1,1$ & 0.0278 \\
2 & $4,5,8,9,13,14,19,21,28,37$ & $1,-1,1,-1,-1,1,-1,1,1,-1$ & 0.0278 \\
3 & $7,8,10,12,29,31$ & $1,-1,-1,1,-1,1$ & 0.0139 \\
4 & $2,3,10,11,26,32$ & $1,-1,1,-1,1,-1$ & 0.0139 \\
5 & $1,3,7,9,27,30$ & $-1,1,-1,1,-1,1$ & 0.0139 \\
6 & $5,6,11,12,14,15,22,23,33,40$ & $1,-1,1,-1,-1,1,1,-1,1,-1$ & 0.0278 \\
7 & $16,17,20,21,35,38$ & $1,-1,1,-1,-1,1$ & 0.0139 \\
8 & $19,20,22,24,39,41$ & $1,-1,-1,1,-1,1$ & 0.0139 \\
9 & $17,18,23,24,36,42$ & $1,-1,1,-1,-1,1$ & 0.0139 \\
\hline
\end{tabular}

Table 9: The triangular surfaces of smoothing domains in node-based smoothing domains

\begin{tabular}{|c|c|c|c|c|c|c|c|}
\hline No. & Vertex & Area & Normal vector & No. & Vertex & Area & Normal vector \\
\hline 1 & $15-8-6$ & 0.0233 & $(0.45,-0.89,0)$ & 31 & $4-17-10$ & 0.0417 & $(1,0,0)$ \\
\hline 2 & $15-9-6$ & 0.0233 & $(-0.45,0.89,0)$ & 32 & $4-19-10$ & 0.0417 & $(-1,0,0)$ \\
\hline 3 & $16-9-6$ & 0.0345 & $(-0.30,-0.90,-0.30)$ & 33 & $1-16-10$ & 0.0417 & $(1,0,0)$ \\
\hline 4 & $16-10-6$ & 0.0345 & $(0.30,0.90,0.30)$ & 34 & $1-17-10$ & 0.0417 & $(-1,0,0)$ \\
\hline 5 & $17-10-6$ & 0.0233 & $(-0.45,0,-0.89)$ & 35 & 5-16-10 & 0.0417 & $(-1,0,0)$ \\
\hline 6 & $17-11-6$ & 0.0233 & $(0.45,0,0.89)$ & 36 & 5-19-10 & 0.0417 & $(1,0,0)$ \\
\hline 7 & $18-8-6$ & 0.0180 & $(-0.58,-0.58,0.58)$ & 37 & $4-17-11$ & 0.0833 & $(0,-1,0)$ \\
\hline 8 & $18-11-6$ & 0.0180 & $(0.58,0.58,-0.58)$ & 38 & 4-18-11 & 0.0833 & $(0,1,0)$ \\
\hline 9 & $19-8-6$ & 0.0329 & $(0,0.95,-0.32)$ & 39 & $2-18-11$ & 0.0833 & $(0,-1,0)$ \\
\hline 10 & $19-10-6$ & 0.0329 & $(0,-0.95,0.32)$ & 40 & $2-20-11$ & 0.0833 & $(0,1,0)$ \\
\hline 11 & $20-9-6$ & 0.0255 & $(0.82,0.41,0.41)$ & 41 & $1-17-11$ & 0.0833 & $(0,1,0)$ \\
\hline 12 & $20-11-6$ & 0.0255 & $(-0.82,-0.41,-0.41)$ & 42 & $1-20-11$ & 0.0833 & $(0,-1,0)$ \\
\hline 13 & $15-9-7$ & 0.0233 & $(0.45,0,-0.89)$ & 43 & $5-15-12$ & 0.0722 & $(0.58,0.58,0.58)$ \\
\hline 14 & $15-12-7$ & 0.0233 & $(-0.45,0,0.89)$ & 44 & $5-21-12$ & 0.0722 & $(-0.58,-0.58,-0.58)$ \\
\hline 15 & $16-9-7$ & 0.0345 & $(0.30,0.30,0.90)$ & 45 & $3-21-12$ & 0.0722 & $(0.58,0.58,0.58)$ \\
\hline 16 & $16-13-7$ & 0.0345 & $(-0.30,-0.30,-0.90)$ & 46 & $3-23-12$ & 0.0722 & $(-0.58,-0.58,-0.58)$ \\
\hline 17 & $20-9-7$ & 0.0255 & $(-0.82,-0.41,-0.41)$ & 47 & $2-15-12$ & 0.0722 & $(-0.58,-0.58,-0.58)$ \\
\hline 18 & $20-14-7$ & 0.0255 & $(0.82,0.41,0.41)$ & 48 & $2-23-12$ & 0.0722 & $(0.58,0.58,0.58)$ \\
\hline 19 & $21-12-7$ & 0.0329 & $(0,0.32,-0.95)$ & 49 & $1-16-13$ & 0.0417 & $(-1,0,0)$ \\
\hline 20 & $21-13-7$ & 0.0329 & $(0,-0.32,0.95)$ & 50 & $1-22-13$ & 0.0417 & $(1,0,0)$ \\
\hline 21 & $22-13-7$ & 0.0233 & $(0.45,0.89,0)$ & 51 & $3-21-13$ & 0.0417 & $(1,0,0)$ \\
\hline 22 & $22-14-7$ & 0.0233 & $(-0.45,-0.89,0)$ & 52 & $3-22-13$ & 0.0417 & $(-1,0,0)$ \\
\hline 23 & $23-12-7$ & 0.0180 & $(0.58,-0.58,0.58)$ & 53 & 5-16-13 & 0.0417 & $(1,0,0)$ \\
\hline 24 & $23-14-7$ & 0.0180 & $(-0.58,0.58,-0.58)$ & 54 & $5-21-13$ & 0.0417 & $(-1,0,0)$ \\
\hline
\end{tabular}




\begin{tabular}{l|c|c|c|c|c|c|c}
25 & $5-15-8$ & 0.0722 & $(-0.58,-0.58,-0.58)$ & 55 & $1-20-14$ & 0.0833 & $(0,0,1)$ \\
26 & $5-19-8$ & 0.0722 & $(0.58,0.58,0.58)$ & 56 & $1-22-14$ & 0.0833 & $(0,0,-1)$ \\
27 & $2-15-8$ & 0.0722 & $(0.58,0.58,0.58)$ & 57 & $2-20-14$ & 0.0833 & $(0,0,-1)$ \\
28 & $2-18-8$ & 0.0722 & $(-0.58,-0.58,-0.58)$ & 58 & $2-23-14$ & 0.0833 & $(0,0,1)$ \\
29 & $4-18-8$ & 0.0722 & $(0.58,0.58,0.58)$ & 59 & $3-22-14$ & 0.0833 & $(0,0,1)$ \\
30 & $4-19-8$ & 0.0722 & $(-0.58,-0.58,-0.58)$ & 60 & $3-23-14$ & 0.0833 & $(0,0,-1)$ \\
\hline
\end{tabular}

Table 10: The node-based smoothing domains

\begin{tabular}{c|c|c|c}
\hline No. & Surfaces & Outer normal vector direction & Vlume \\
\hline 1 & $3,4,5,6,11,12,15,16,17,18,21,22$, & $-1,1,-1,1,1,-1,1,-1,-1,1,1,-1$, & 0.0417 \\
& $33,34,41,42,49,50,55,56$ & $-1,1,-1,1,1,-1,-1,1$ & \\
2 & $1,2,7,8,11,12,13,14,17,18,23,24$, & $-1,1,1,-1,-1,1,-1,1,1,-1,-1,1$, & 0.0417 \\
& $27,28,39,40,47,48,57,58$ & $1,-1,1,-1,-1,1,1,-1$ & \\
3 & $19,20,21,22,23,24,45,46,51,52,59$, & $-1,1,-1,1,1,-1,1,-1,-1,1,-1$, & 0.0208 \\
& 60 & 1 & \\
4 & $5,6,7,8,9,10,29,30,31,32$, & $1,-1,-1,1,1,-1,1,-1,-1,1$, & 0.0208 \\
& 37,38 & $1,-1$ & \\
5 & $1,2,3,4,9,10,13,14,15,16,19,20$, & $1,-1,1,-1,-1,1,1,-1,-1,1,1,-1$, & 0.0417 \\
& $25,26,35,36,43,44,53,54$ & $-1,1,1,-1,1,-1,-1,1$ & \\
\hline
\end{tabular}

\section{2 Example 2: A rectangular bar}

As the second example we consider the bar shown in Figure 7. Based on the original mesh with 12, 20 and 36 T4 elements (Figure 7), we construct the face-based, edge-based and node-based smoothing domains for FS-FEM, ES-FEM and NS-FEM models respectively. In Table 11, we present the number surfaces of smoothing domains for different kinds of models.

Table 11: The number of surfaces of smoothing domains for different S-FEM models

\begin{tabular}{c|c|c|c}
\hline \multirow{2}{*}{$\begin{array}{c}\text { The number of elements } \\
\text { in original mesh }\end{array}$} & \multicolumn{3}{|c}{ The number of triangular surfaces of smoothing domains in S-FEM mesh } \\
\cline { 2 - 4 } & FS-FEM model & ES-FEM model & NS-FEM model \\
\hline 12 & 92 & 204 & 264 \\
20 & 180 & 396 & 504 \\
36 & 356 & 780 & 984 \\
\hline
\end{tabular}




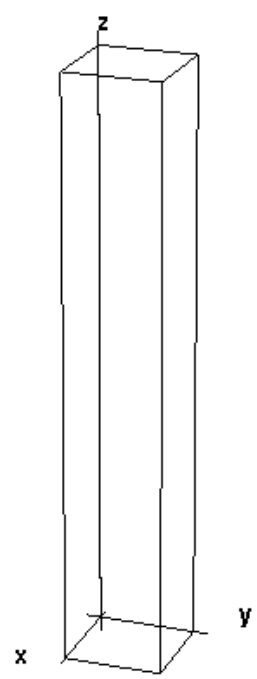

(a) bar

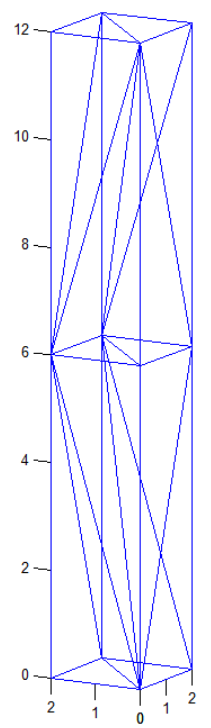

(b) 12 elements

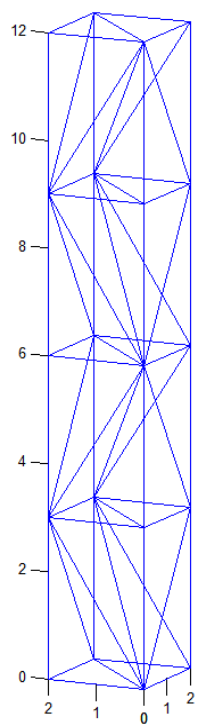

(c) 20 elements

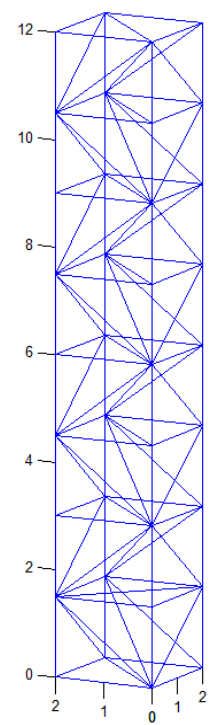

(d) 36 elements

Figure 7: A bar meshed with different number of T4 elements

\section{3 Example 3: A rectangular bar with a cylinder}

A more complicated example is for the domain of a rectangular bar with a cylinder as shown in Figure 8. The original T4 mesh we used has 583 nodes, 3047 edges, 4411 faces and 1947 elements. Then the constructed node-based, edge-based and face-based smoothing domains contain 23814, 2358 and 11757 triangular surfaces respectively.

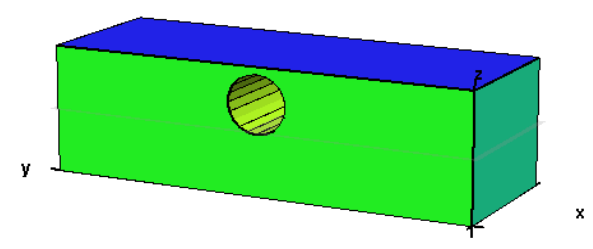

Figure 8: A rectangular bar with a cylinder

\section{Conclusion}

In S-FEM, it is necessary to establish smoothing domains based on a background mesh covering the problem domain. One smoothing doma in may include not only one element of the original mesh but also its neighboring elements. The S-FEM models can be node-based, edge-based, face-based and cell-based. Hence the twelve connectivity lists among nodes, edges, faces and elements are required. As S-FEM works well for T4 elements, we present an efficient approach for creating smoothing domains using the robust and easy-to-use software TetGen for generating T4 elements. Our program can automatically generate the twelve connectivity lists, including the connectivity of F-N, F-Eg, F-Ele, N-F, Eg-F, Ele-F and those we have introduced 
previously for 2D problems [1]. People usually use a volume-weighted average of strains calculated by FEM on the neighboring elements as the smoothed strains because it will not be easy to create smoothing domains and do surface integralalong the surface of smoothing domains, especially in 3D case. This paper is the first time to present a comprehensive procedure and efficient algorithms for creating smoothing domains systematically with all the necessary information required for the surface-integral, including the volume of smoothing domains and the area and the outer normal vector of surfaces. The algorithms given in this paper have been successfully tested on different problem domains. The algorithms enable the automation in computation and adaptive analys is of 3D solids using S-FEM working well with the T4 elements that can be generated automatically.

Please note that we can also use mesh handling frameworks like Mesh-Oriented datABase (MOAB) [38] and work in [39] to generate or store additional mesh related data, when need using the concepts of smoothing domains in S-FEM.

\section{Acknowledgments}

This basic research by the senior author is partially sponsored by NSF under the Award No. DMS-1214188. The work by all the authors is partially supported by the National Natural Science Foundation of China (Grant No. 11472184). The authors would like to thank the valuable support leading to this paper.

\section{Reference}

[1] Y. LI, M. LI and G R. Liu, A modified triangulation algorithm tailored for the smoothed finite element method (S-FEM), International journal of numerical methods, Vol. 11, No.1 (2014) 1350069.

[2] Liu GR., Dai KY, Nguyen TT, A smoothed finite element method for mechanics problems, Comput Mech, 2007 39: 859-877.

[3] Liu GR., T. T. Nguyen, K. Y. Dai and K. Y. Lam, Theoretical aspects of the smoothed finite element method (S-FEM), Int. J. Numer. Meth. Engng. 2007; 71: 902-930.

[4] Dai, K. Y., Liu GR. and T. T. Nguyen. An n-sided polygonal smoothed finite element method (nS-FEM) for solid mechanics. Finite Elements in Analysis and Design 2007; 43: 847-860.

[5] Liu GR and Nguyen-Thoi T, Smoothed Finite Element Methods, CRC Press: Boca Raton, USA, 2010.

[6] Zhang, Z., Liu, G R., \& Khoo, B. C. (2012). Immersed smoothed finite element method for two dimensional fluid-structure interaction problems. International Journal for Numerical Methods in Engineering, 90(10), 1292-1320. doi: 10.1002/nme.4299

[7] Dai KY, Liu GR. Free and forced analysis using the smoothed finite element method (SFEM). J Sound Vib 2007; 301:803-820. 
[8] Cui, X. Y., Liu, G R., Li, G Y., Zhang, G Y., \& Sun, G Y. (2009). Analysis of elastic-plastic problems using edge-based smoothed finite element method. International Journal of Pressure Vessels and Piping, 86(10), 711-718.

[9] Zhang, Z., Liu, G R., \& Khoo, B. C. (2013). A three dimensional immersed smoothed finite element method (3D IS-FEM) for fluid-structure interaction problems. Computational Mechanics, 51(2), 129-150. doi: 10.1007/s00466-012-0710-1

[10] Liu GR. 2009. A G space theory and weaken weak $\left(W^{2}\right)$ form for a unified formulation of compatible and incompatible methods, Part I: Theory and Part II :Applications to solid mechanics problems. International Journal for Numerical Methods in Engineering; doi:10.1002/nme.2719 and doi:10.1002/nme.2720.

[11] Liu GR. 2008. A generalized gradient smoothing technique and the smoothed bilinear form for Galerkin formulation for a wide class of computational methods. International Methods of Computational Methods; 5(2): 199-236.

[12] Liu GR. 2009. On and Zhang GY. 2009. A normed G space and weakened weak (W2) formulation of a cell-based Smoothed Point Interpolation Method. International Journal of Computational Methods; 6(1): 147-179.

[13] Liu GR. 2009. On the G space theory. International Journal of Computational Methods; 6(2): 257-289

[14] G R. Liu, G Y. Zhang. Smoothed Point Integration Methods: G Space theory and Weakened Weak Forms; World Scientific Publishing Co. Pte. Ltd.

[15] Zhang, Z., \& Liu, G R. (2011). An edge-based smoothed finite element method (ES-FEM) using 3-node triangular elements for 3D non-linear analysis of spatial membra ne structures. International Journal for Numerical Methods in Engineering, 86(2), 135-154. doi: 10.1002/nme.3049

[16] Liu, G R., Chen, L., Nguyen-Thoi, T., Zeng, K. Y., \& Zhang, G Y. (2010). A novel singular node-based smoothed finite element method (NS-FEM) for upper bound solutions of fracture problems. International Journal for Numerical Methods in Engineering, 83(11), 1466-1497.

[17] Chen JS, Wu CT, Yoon Y. A stabilized conforming nodal integration for Galerkin mesh-free methods. Int J Numer Methods Engrg 2001;50:435-466.

[18] Liu GR, Nguyen-Thoi T, Lam KY. A node-based smoothed finite element method for upper bound solution to solid problems (NS-FEM). Comput Struct 2008;87:14-26.

[19] Liu GR, Nguyen-Thoi T, Lam KY. An edge-based smoothed finite element method (ES-FEM) for static, free and forced vibration analysis. J Sound Vib 2009;320:1100-1130.

[20] Liu GR., Dai KY, Nguyen TT, A smoothed finite element method for mechanics problems, Comput Mech, 2007 39: 859-877.

[21] T. Nguyen-Thoi, G R. Liu, K. Y. Lam and G Y. Zhang. A face-based smoothed finite element method (FS-FEM) for 3D liner and geometrically non-linear solid mechanics problems using 4-node tetrahedral elements. International Journal of Numerical Methods in Engineering. 2009, 78:324-353.

[22] Bathe KJ. Finite Element Procedures. MIT Press, Prentice-Hall: Cambridge. MA, 
Englewood Cliffs, NJ, 1996.

[23] Liu GR, Quek SS. The Finite Element method: A Practical Course. Butterworth Heinemann: Oxford, 2003.

[24] Zienkiewicz OC, Taylor RL. The finite Element Method ( $5^{\text {th }}$ edn), Butterworth Heinemann: Oxford, 2000.

[25] Liu GR and Nguyen-Thoi T, Smoothed Finite Element Methods, CRC Press: Boca Raton, USA, 2010.

[26] Liu GR, Nguyen-Thoi T, Lam KY. A node-based smoothed finite element method for upper bound solution to solid problems (NS-FEM). Comput Struct 2008;87:14-26.

[27] Liu GR, Nguyen-Thoi T, Lam KY. An edge-based smoothed finite element method (ES-FEM) for static, free and forced vibration analys is. J Sound Vib 2009;320:1100-1130.

[28] Dai, K. Y., Liu GR. and T. T. Nguyen. An n-sided polygonal smoothed finite element method (nS-FEM) for solid mechanics. Finite Elements in Analysis and Design 2007; 43: 847-860.

[29] D. T. Lee and A. K. Lin. Generalized Delaunay triangulation for planar graphs. Discrete and Computational Geometry, 1:201-217, 1986.

[30] J. R. Shewchuk. A condition guaranteeing the existance of higher-dimensional constrained Delaunay triangulations. In Proc. $14^{\text {th }}$ Ann. Symp. On Comput. Geom., pages 76-85, 1998.

[31] J. R. Shewchuk. general-dimensional constrained Delaunay and constrained regular triangulations, i: combinatorial properties. Discrete and Computational Geometry, 39:580-637, 2008.

[32] J. Ruppert. A Delaunay refinement algorithm for quality 2-dimensional mesh generation. Journal of Algorithms, 18(3):548-585, 1995.

[33] J. R. Shewchuk. Tetrahedral mesh generation by Delaunay refinement. In Proc. $14^{\text {th }}$ Ann. Symp. On Comput. Geom., pages 86-95, 1998.

[34]H. Si and K Gaertner, 3d boundary recovery by constrained Delaunay tetrahedralization. International Journal for Numerical Methods in Engineering, 85:1341-1364, 2011.

[35] G L. Miller, D. Talmor, S.-H. Teng, N. J. Walkington, and H. Wang. Control volume meshes using sphere packing: Generation, refinement and coarsening. In Proc. $5^{\text {th }}$ Intl. Meshing Roundtable, 1996.

[36] TetGen, A Quality Tetrahedral Mesh Generator and 3D Delaunay Triangulator. Version 1.5, User's Manual.

[37] J.S. Chen, C.T. Wu, S. Yoon, Y.You, A stabilized conforming nodal integration for Galerkin mesh-free methods. International Journal for Numerical Methods in Engineering. 50:435-466, 2001.

[38] TJ Tautges, C Ernst, C Stimpson, RJ Meyers and K Merkley, MOAB: a mesh-oriented database, Sandia National Laboratories, 2004.

[39]G Berti, Generic Software Components for Scientific Computing,PhD Dissertation, TU Cottbus, 2000. 